\title{
Relationships between muscular impairments, pain, and disability in patients with chronic nonspecific low back pain: a cross sectional study
}

\author{
Ali Bozorgmehr', Shahla Zahednejad², Reza Salehi ${ }^{3}$ Noureddin Nakhostin Ansar', Soheila Abbasi', Holakoo Mohsenifar', \\ Jorge Hugo Villafañe $e^{5, *}$ \\ 'Department of Physical Therapy, School of Rehabilitation Sciences, Iran University of Medical Science, Tehran, Iran \\ ${ }^{2}$ School of Rehabilitation Sciences, Student Research Committee, Musculoskeletal Rehabilitation Research Center, Ahvaz Jundishapur University of Medical Sciences, \\ Ahvaz, Iran \\ ${ }^{3}$ Rehabilitation Resarch Center, Department of Rehabilitation Management, School of Rehabilitation Sciences, Iran University of Medical Sciences, Tehran, Iran \\ ${ }^{4}$ Department of Physiotherapy, School of Rehabilitation Science, Tehran University of Medical Science, Tehran, Iran \\ ${ }^{5}$ RCCS Fondazione Don Carlo Gnocchi, Milan, Italy
}

To clarify the significance of mechanical impairments, pain, and functional limitations as predictors of chronic low back pain (LBP). Ninety patients with chronic nonspecific low back pain (CNSLBP) were prospectively studied with clinical tests and questionnaires. Changes in muscle extensibility and endurance tests were evaluated and changes assessed in LBP intensity on numeric rating scale $0-10$ and severity with Oswestry Disability Index (ODI) 0-100. In the present study we found significant associations between the 4 muscle extensibility and 2 endurance tests and pain at nonspecific patients with chronic LBP $(P<0.005)$. The 2 muscle extensibility and 1 endurance tests were in complete equilibrium with ODI disability and hence showed similar results $(P<0.005)$. The associations between the muscle extensibility and endurance tests and pain were significantly elevated in patients with nonspecific chronic LBP.

Keywords: Chronic low back pain, Disability, Endurance

\section{INTRODUCTION}

Chronic nonspecific low back pain (CNSLBP) has been defined as a persistent pain in the lower back for at least three months with no known causes (Ferrari et al., 2015; Isgro et al., 2014; Lee and Kang, 2016). The CNSLBP prevalence is estimated of $90 \%$ among all types of the low back pain (LBP) (Maniadakis and Gray, 2000; Page et al., 2002; Rubin, 2007). The LBP imposes high direct and indirect costs on the patients and the society (Henschke et al., 2008; Hestbaek et al., 2003). Whereas a specific pathology has not been identified for CNSLBP, mechanical factors (e.g., changes in muscle length, strength, or endurance) may contribute to the pain and disability (Bae et al., 2017; Farahbakhsh et al., 2018; Nourbakhsh and Arab, 2002; Pillastrini et al., 2015; Villa- fañe et al., 2015; Villafañe et al., 2016; Yoon and Park, 2013).

Previous studies have attempted to measure the relationship between mechanical factors and LBP prevalence (Bayramoğlu et al., 2001). Authors reported that decreased endurance of back muscles and strength of pelvic muscles were closely correlated with the severity of LBP (Nourbakhsh and Arab, 2002). Despite the presence of various mechanical impairments in patients with CNSLBP, the relationship among mechanical impairments, pain and functional limitations in patients with CNSLBP has not been properly investigated (Negrini et al., 2013). This study sought to determine the relationship among muscular impairments, pain, and disability in patients with CNSLBP and to clarify the significance of muscular impairments, pain, and disability as predictors of CNSLBP.
${ }^{*}$ Corresponding author: Jorge Hugo Villafañe (iD https://orcid.org/0000-0002-3239-7626 IRCCS Fondazione Don Carlo Gnocchi, Milan, Italy

Tel: +39-339-5857563, E-mail: mail@villafane.it

Received: August 1, 2018 / Accepted: September 29, 2018
This is an Open Access article distributed under the terms of the Creative Commons Attribution Non-Commercial License (http://creativecommons.org/licenses/by-nc/4.0/) which permits unrestricted non-commercial use, distribution, and reproduction in any medium, provided the original work is properly cited. 


\section{MATERIALS AND METHODS}

\section{Study design}

A cross-sectional study design was used. Informed consent was obtained from all patients, and procedures were conducted according to the Declaration of Helsinki. The study protocol was approved by the Ethics Committee of Ahvaz Jundishapur University of Medical Sciences, Ahvaz, Iran (IR.AJUMS.REC.1391.476). The patients were asked to sign an informed consent form before participation. The study has been registered at Trial Registration Current Controlled Trials website (NCT02932163).

\section{Subjects}

Patients with chronic nonspecific LBP were recruited from five hospitals in Ahvaz, Iran. Following inclusion criteria were used: (a) LBP duration $\geq 6$ months and (b) pain intensity $\geq 2$ on the $0-10$ Numerical Rating Scale (NRS).

Exclusion criteria: Patients with other orthopedic or neurological disorders (e.g., spinal osteoarthritis, spondylolisthesis, history of vertebral fractures) and the patient with the back surgery and leg pain were excluded (Pillastrini et al., 2013).

To calculate the minimum required sample size, a confidence level of $95 \%$, the power of study $80 \%$ and the average prevalence rate of low back pain $80 \%$ was considered. This calculation generated a sample size of 85 individuals.

\section{Outcome measures}

The outcome measures adopted were the NRS, the Oswestry Disability Questionnaire in its Persian version (ODI-P), the muscle extensibility, the Static Flex Endurance Test (SFET), and the Static Side Bridge Endurance Test (SSBET).

\section{Numerical Rating Scale}

A 0-10 NRS was administered to evaluate pain intensity. The participants were asked to indicate their pain intensity from zero (no pain) to 10 (worst pain) (Parazza et al., 2014).

\section{Oswestry Disability Questionnaire}

The Oswestry Disability Questionnaire is a widely used instrument to measure disability in patients with LBP. It consists of ten sections with a total possible score ranging from 0 to 50 (Fairbank et al., 1980). Total percentage scores range between 0 and 100 and they are categorized as follow: $0 \%-20 \%=$ minimal disability; $21 \%-$ $40 \%=$ moderate disability; $41 \%-60 \%=$ severe disability; $61 \%-$ $80 \%=$ crippled; $81 \%-100 \%$ = bed bound (Fairbank et al., 1980)
The validated ODI-P was used in this study (Mousavi et al., 2006).

\section{Muscle extensibility tests}

A standard goniometer was utilized to measure the extensibility of rectus femoris, hip lateral rotators, tensor fasciae latae, and hamstring muscles. Previous investigation has found intratester interclass correlation coefficients for all motions ranging from 0.87 to 0.99 , and the degree of inter-tester reliability for these measurements appears to be range-of-motion specific (Riddle et al., 1987).

Rectus femoris muscle: The subject was in a supine position, knee flexed over the edge of the bed. The axis of the goniometer was placed over the lateral femoral epicondyle, the stationary arm was parallel to the long axis of the femur, and the movable arm was parallel to the long axis of the fibula. The end point of the active knee flexion was based on discomfort reported by patients.

Hip lateral rotators muscles group (piriformis, gemellus superior, obturator intenus, gemellus inferior, quadratus femoris, and obturator externus: The subject was in the prone position with one knee flexed at $90^{\circ}$, and the pelvis firmly stabilized through strapping. The axis of the goniometer was placed over the tibial tuberosity. While the stationary arm was kept in place perpendicular to the floor, the movable arm parallel to the anterior aspect of the tibia was moved with the leg in a medial direction to the limit of lateral rotation. The end position was recorded.

Tensor fasciae latae muscle: The subject was in the supine position with the not-tested hip in flexion and the tested leg hanging off the bed. The axis of the goniometer was placed over the anterior superior iliac spine (ASIS) of the tested leg, the stationary arm along a line connecting both ASISs, and the movable arm was parallel to the axis of the femur. The tested leg was then adducted to the limit of range of motion and the end position was recorded.

Hamstring muscles: The subject was in the supine position. The axis of the goniometer was placed over the greater trochanter, the stationary arm was parallel to the lateral trunk, and the movable arm was parallel to the axis of the femur. The patient's hip was then passively flexed, always maintaining knee extension. The end position of hip flexion was recorded.

\section{Endurance tests}

Static Flex Endurance Test: The SFET was used to assess the endurance of trunk flexor muscles. The SFET was performed with patient in supine, crook lying position (Vanti et al., 2017). The subject was then asked to flex his/her trunk, touch his/her knees, and hold this position as long as possible (Fig. 1A).

Static Side Bridge Endurance Test: The endurance of lateral trunk 

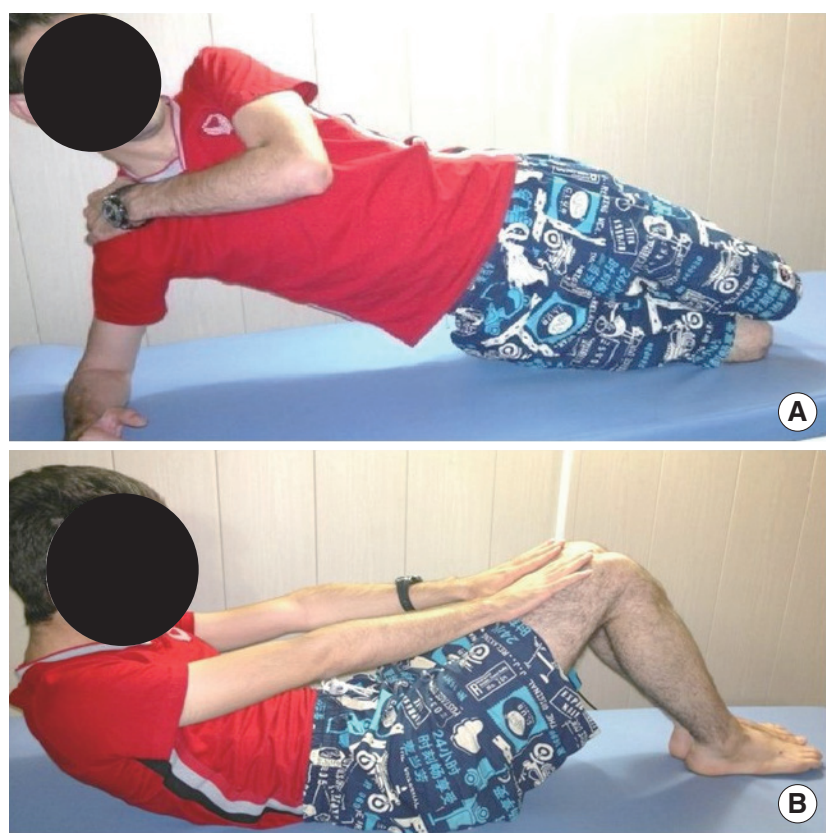

Fig. 1. Static flex endurance test and static side bridge endurance test. (A) Static flex endurance test (SFET). The SFET was performed with patient in supine, crook lying position. The subject was then asked to flex his/her trunk, touch his/her knees, and hold this position as long as possible. (B) Static side bridge endurance test (SSBET). The SSBET was performed in side lying position on a plinth with resting on a pronated forearm with the elbow placed under shoulder level. The legs were together, knees bent to $90^{\circ}$, and hips flexed no more than 20. Subject was then asked to support his/her weight only on the lower elbow and feet while lifting his/her hips off the plinth. The test was stopped when hips returned to the mat. Informed consent was obtained from the subject for the publication.

flexor muscles was assessed using the SSBET. The SSBET was performed in side lying position on a plinth with resting on a pronated forearm with the elbow placed under shoulder level. The legs were together, knees bent to $90^{\circ}$, and hips flexed no more than $20^{\circ}$ (Vanti et al., 2017). The subject was then asked to support his/her weight only on the lower elbow and feet while lifting his/ her hips off the plinth. The test was stopped when hips returned to the mat (Fig. 1B).

\section{Protocol}

Demographic data including height, age, gender, and LBP duration were recorded first. Patients were asked to indicate their pain intensity and LBP related disability using NRS and ODI, respectively. The muscular extensibility was measured using a standard goniometer. Finally, the SFET and the SSBET were used to measure the muscle endurance of trunk flexors and trunk lateral flexors, respectively. A physiotherapist with 8 years of therapeutic experience performed all the measurements. The trial was de-
Table 1. Descriptive statistics and functional scores of the cohort $(n=90)$

\begin{tabular}{|c|c|c|}
\hline Parameter & Mean \pm SD & Range \\
\hline Height (cm) & $173.3 \pm 8.35$ & 150-186 \\
\hline Weight (cm) & $77.4 \pm 11.2$ & 49-98 \\
\hline Age (yr) & $29.5 \pm 8.0$ & $20-47$ \\
\hline Female gender, n (\%) & 20 (22.2) & - \\
\hline Pain duration (mo) & $7.6 \pm 2.1$ & $4-12$ \\
\hline Pain intensity (0-10) & $3.4 \pm 1.1$ & $2-6$ \\
\hline ODI (0-100) & $21.2 \pm 4.9$ & $12-34$ \\
\hline $\operatorname{RF} \cdot \mathrm{L}\left({ }^{\circ}\right)$ & $42.4 \pm 3.0$ & $35-47$ \\
\hline $\operatorname{RF} \cdot R\left({ }^{\circ}\right)$ & $42.3 \pm 3.7$ & $24-46$ \\
\hline HMS.L $\left(^{\circ}\right)$ & $85.1 \pm 8.2$ & $60-95$ \\
\hline $\mathrm{HMS} . \mathrm{R}\left({ }^{\circ}\right)$ & $85.3 \pm 7.8$ & $61-95$ \\
\hline LRs.L $\left(^{\circ}\right)$ & $30.8 \pm 5.0$ & $19-40$ \\
\hline $\operatorname{LRs} . \mathrm{R}\left(^{\circ}\right)$ & $30.7 \pm 4.7$ & $20-40$ \\
\hline TFL.L $\left(^{\circ}\right)$ & $54.1 \pm 8.4$ & $35-66$ \\
\hline TFL.R $\left(^{\circ}\right)$ & $53.7 \pm 8.0$ & $36-66$ \\
\hline SFET (sec) & $21.3 \pm 4.9$ & $12-37$ \\
\hline SSET.L (sec) & $55.9 \pm 9.9$ & $35-80$ \\
\hline SSET.R (sec) & $57.2 \pm 9.2$ & 30-78 \\
\hline
\end{tabular}

SD, standard deviation; ODI, Oswestry Disability Index; RF.L, rectus femoris left; RF.R, rectus femoris right; HMS.L, hamstring left; HMS.R, hamstring right; LRs.L, lateral rotators left; LRs.R, lateral rotators right; TFL.L, tensor fascialata left; TFL.R, tensor fascialata right; SFET, static flexion endurance test; SSET.L, static side bridge endurance test left; SSET.R, static side bridge endurance test right.

signed according to the STROBE publishing guidelines.

\section{Statistical analysis}

Data were analyzed using IBM SPSS Statistics ver. 22.0 (IBM Co., Armonk, NY, USA). Descriptive statistics were provided for all subjects, as well as for ODI-I, NRS, muscle extensibility tests and endurance tests, respectively. A linear regression model was used to determine the relationship between the muscular impairments, pain, and disability. A level of significance at 0.05 was used for the statistical analyses.

\section{RESULTS}

\section{Clinical characteristics of the participants}

A total of 90 consecutive subjects, $22.2 \%$ female $(29.5 \pm 8.0$ years) with an ODI-I that on average included $21.2 \pm 4.9$ and pain intensity $3.4 \pm 1.1$ were included in the study (Table 1). No adverse effects were detected during or after the measurements.

\section{Pain intensity and related disability measurements of endurance and muscle extensibility}

The Pearson correlation analysis demonstrated that muscle ex- 
tensibility parameter as hamstring left-right (HMS.L-R), lateral rotators left-right (LR.L-R) and tensor fascialata right (TFL.R) was significantly negative correlated with pain intensity $(r=-0.69$ to $-0.84, P<0.001)$. The pain intensity was negatively correlated with endurance tests parameter as static side bridge endurance test (SSET) $(r=-0.56)$, SSET left (SSET.L) $(r=-0.59)$ and SSET right (SSET.R) $(r=-0.53)$ with $P<0.001$ (Table 2). ODI disability parameters were negatively correlated with the endurance parameters (HMS.L, HMS.R, and LR.R) with $P<0.04$ and muscle exten- sibility parameters (SSER.L and SSET.R) with $P<0.007$ ) (Table 2).

\section{Endurance and muscle extensibility association analysis}

Table 3 presents the factors associated with the pain in nonspecific LBP patients. In multiple linear regression analysis, lateral rotators left (LRs.L), rectus femoris left (RF.L), TFL.R, STSF.TL, and SFET tests were associated with the pain (standardized coefficient $\beta=-0.15$ to -0.58 , all $P=0.001$ ) in nonspecific LBP patients. In the model with ODI as a dependent variable, HMS.L, RF.L, and

Table 2. The Pearson correlations between pain severity, disability, and muscular variables

\begin{tabular}{|c|c|c|c|c|c|c|c|c|c|c|c|}
\hline Variable & RF.L & RF.R & HMS.L & HMS.R & LRs.L & LRs.R & TFL.L & TFL.R & SFET & SSET.L & SSET.R \\
\hline \multicolumn{12}{|l|}{ Pain intensity } \\
\hline Pearson correlation & -0.06 & -0.04 & $-0.72^{* *}$ & $-0.69^{* *}$ & $-0.84^{* *}$ & $-0.78^{* *}$ & $-0.84^{* *}$ & $-0.83^{* *}$ & $-0.56^{* *}$ & $-0.59^{* *}$ & $-0.53^{* *}$ \\
\hline$P$-value & 0.57 & 0.71 & 0.001 & 0.001 & 0.001 & 0.001 & 0.001 & 0.001 & 0.001 & 0.001 & 0.001 \\
\hline \multicolumn{12}{|l|}{ Disability (ODI) } \\
\hline Pearson correlation & $-0.23^{*}$ & -0.14 & $-0.25^{*}$ & $-0.25^{*}$ & -0.20 & $-0.22^{*}$ & -0.13 & -0.12 & -0.05 & $-0.28^{* *}$ & $-0.28 * *$ \\
\hline$P$-value & 0.03 & 0.20 & 0.018 & 0.020 & 0.058 & 0.040 & 0.232 & 0.275 & 0.616 & 0.007 & 0.008 \\
\hline
\end{tabular}

RF.L, rectus femoris left; RF.R, rectus femoris right; HMS.L, hamstring left; HMS.R, hamstring right; LRs.L, lateral rotators left; LRs.R, lateral rotators right; TFL.L, tensor fascialata left; TFL.R, tensor fascialata right; SFET, static flexion endurance test; SSET.L, static side bridge endurance test left; SSET.R, static side bridge endurance test right; ODI, Oswestry Disability Index.

${ }^{*}$ Correlation is significant at the 0.05 level. ${ }^{* *}$ Correlation is significant at the 0.01 level (two-tailed).

Table 3. Factors affecting pain severity using linear regression

\begin{tabular}{lcccccc}
\hline Variable & B & SE & Beta & $t$ & $P_{\text {-value* }}$ & 95\% Confidence interval \\
\hline Muscle extensibility tests & & & & & & \\
$\quad$ Constant & 12.567 & 0.590 & - & 21.142 & $<0.001$ & 11.385 to 13.749 \\
TFL.L & 0.011 & 0.021 & 0.085 & 0.513 & 0.610 & -0.032 to 0.054 \\
LRs.L & -0.121 & 0.011 & -0.559 & -10.802 & $<0.001$ & -0.144 to -0.099 \\
RF.L & -0.043 & 0.011 & -0.149 & -3.879 & $<0.001$ & -0.066 to -0.021 \\
TFL.R & -0.076 & 0.021 & -0.578 & -3.704 & $<0.001$ & -0.121 to -0.036 \\
Endurance tests & & & & & \\
Constant & 7.704 & 0.520 & - & 14.809 & $<0.001$ & 6.670 to 8.738 \\
SSET.L & -0.046 & 0.010 & -0.421 & -4.758 & $<0.001$ & -0.065 to -0.027 \\
SFET & -0.082 & 0.019 & -0.372 & -4.201 & $<0.001$ & -0.120 to -0.043 \\
\hline
\end{tabular}

SE, standard error; RF.L, rectus femoris left; LRs.L, lateral rotators left; TFL.L, tensor fascialata left; TFL.R, tensor fascialata right; SFET, static flexion endurance test; SSET.L, static side bridge endurance test left.

Table 4. Factors affecting disability level using linear regression

\begin{tabular}{lcccccc}
\hline Variable & B & SE & Beta & $t$ & $P_{\text {-value }}^{*}$ & $95 \%$ Confidence interval \\
\hline Muscle extensibility tests & & & & & & \\
$\quad$ Constant & 50.175 & 8.781 & - & -7.714 & $<0.001$ & 32.723 to 67.627 \\
HMS.L & -0.152 & 0.061 & -0.252 & -2.499 & 0.014 & -20.273 to -0.031 \\
RF.L & -0.380 & 0.166 & -0.231 & -2.290 & 0.024 & -0.710 to -0.050 \\
Endurance tests & & & & & \\
Constant & 28.979 & 2.877 & - & 10.074 & $<0.001$ & 23.263 to 34.969 \\
STSF.TL & -0.140 & 0.051 & -0.282 & -2.762 & 0.007 & -0.241 to -0.039 \\
\hline
\end{tabular}

SE, standard error; RF.L, rectus femoris left; HMS.L, hamstring left; SSET.L, static side bridge endurance test left. 
SSET.L tests were still associated with the disability (standardized coefficient $\beta=-0.23$ to $-0.28, P=0.007$ to 0.024 ) as well (Table 4).

\section{DISCUSSION}

In the present study we found significant associations between the LRsL, RF.L, TFL.R, SSET.L, and SFET tests and pain at nonspecific patients with chronic LBP. The HMS.L, RF.L, and SSET.L tests were in complete equilibrium with ODI disability and hence showed similar results. Relationship between severity of pain and disability appeared as significant, whereas no relationship emerged from amount of pain and disability. This is typical for a chronic condition, characterized by more significant disability than pain.

The extensibility of the tensor fasciae latae and lateral rotators had significant correlation with negative direction with pain severity. As tensor fasciae latae attached to the ilium, reduction in its extensibility leads to anterior innominate rotation and lateral pelvic tilt. In fact, since muscles of the pelvic region work as a group, any muscular imbalance in this region has an impact on other muscles (Langella et al., 2017). This pattern of muscular imbalance results in lateral pelvic rotation and exerts pressure on the elements of the back, e.g., facet joints and soft tissue (Nourbakhsh and Arab, 2002). According to Nourbakhsh and Arab (2002) since a shortened tensor fasciae latae muscle causes lateral pelvic rotation and an altered lumbo-pelvic movement pattern.

Our results in line with this statement "negative effect of pain on muscular activity and confirm that time-dependent changes in soft tissue because of creep and fatigue can lead to alteration in the sensorimotor function of muscles and disorder in the ability of a muscle to protect the soft tissue from injury" that mention in work of Pillastrini et al. (2016) and Vanti et al. (2016).

While some researchers consider functional impairment of one group of muscles in the lumbo-pelvic region to be key factor in the development of LBP, others like Langella et al. (2017) and Bissolotti et al. (2014) have introduced muscular imbalance in the lumbo-pelvic region as the most important factor contributing to nonspecific chronic LBP. They suggested specific and predictable patterns of impairment in the lumbo-pelvic region, i.e., particular muscular imbalance patterns in the pelvic girdle, to be responsible for chronic LBP.

In the current study, reduced muscular extensibility was not significantly related with functional disability. Substantial reductions in muscular flexibility are not present in patients without severe functional disability (Kuukkanen and Mälkiä, 2000). Our finding is thus reasonable since our participants did not have high rates of extensibility reduction or disability. Kuukkanen and Mälkiä (2000) stated that improving muscle flexibility could not effectively treat LBP in patients without severe functional disability. However, there was no correlation between ODI-P disability and the flexibility of muscles. Moreover, higher levels of pain will be associated with increased disability. Philips and Grant (1991) reported chronic LBP to exert the greatest effects on the worsening of disability.

Muscular endurance is recognized as a very important factor in patients with LBP. In fact, getting tired will prevent the patients from performing their daily activities using the involved muscles. Since trunk muscles are active in almost every activity (even in sitting or standing positions or while rolling over), they should be able to work without getting fatigued throughout the day. Moffroid (1997) highlighted muscle fatigue and pain as the dominant complaint of patients with LBP. Therefore, a lack of muscular endurance can play a major role in the development of movement disorders in patients with LBP. According to Hodges (2003), muscular function, especially in case of postural and deep muscles, is impaired in patients with chronic LBP. Such impairment and weakness of the postural muscles involved in the lateral flexion of the trunk (segments of internal oblique and quadratus lumborum) will in turn contribute to the progression of LBP in the patients. The results of the current study also revealed a moderate relationship between pain severity and endurance of these muscles and did not find any significant correlation between endurance and disability level. The ODI-P includes several functional activities requiring constant contraction of these muscles (Fairbank et al., 1980). Since increased severity of LBP is associated with more disability (Philips and Grant, 1991), the endurance of the mentioned muscles can also be effective on the ODI. Compared to deep muscles, the functionality of flexor muscles of the trunk which are responsible for high-torque movements (a characteristic of global muscles according to Hodges), will be less impaired (Philips and Grant, 1991). They will hence be less inefficient in comparison to the local muscles (Moffroid, 1997).

Since these findings might have been caused by the participants' low levels of disability or the absence of a categorization process, future studies are recommended to evaluate a wider range of musculoskeletal disorders and higher levels of pain and disability in patients with LBP. In addition, more accurate results may be obtained by categorizing the patients based on magnetic resonance imaging and new approaches to motion control. Designing treatment protocols and reevaluating the patients during the course of treatment can further refine the findings. Since the va- 
lidity of functional disability indices in patients with LBP (whose major complaints involve functional disability) has been previously confirmed, more attention must to be paid to the recognition, assessment, and treatment of movement disorders with mechanical causes.

In our method for assessment of muscular extensibility, we have to consider some confounding variables such as joint mobility, arthritis and joint dysfunctions, because this factor effective in the end range barrier.

Based on our findings, duration of pain and lumbar disability are significantly related; muscular extensibility and endurance appeared affected by the severity of pain.

\section{CONFLICT OF INTEREST}

No potential conflict of interest relevant to this article was reported.

\section{REFERENCES}

Bae HI, Kim DY, Sung YH. Effects of a static stretch using a load on low back pain patients with shortened tensor fascia lata. J Exerc Rehabil 2017;13:227-231

Bayramoğlu M, Akman MN, Kilinç S, Cetin N, Yavuz N, Ozker R. Isokinetic measurement of trunk muscle strength in women with chronic low-back pain. Am J Phys Med Rehabil 2001;80:650-655.

Bissolotti L, Gobbo M, Villafañe JH, Negrini S. Spinopelvic balance: new biomechanical insights with clinical implications for Parkinson's disease. Eur Spine J 2014;23:576-583.

Fairbank JC, Couper J, Davies JB, O'Brien JP. The Oswestry low back pain disability questionnaire. Physiotherapy 1980;66:271-273.

Farahbakhsh F, Akbari-Fakhrabadi M, Shariat A, Cleland JA, Farahbakhsh F, Seif-Barghi T, Mansournia MA, Rostami M, Kordi R. Neck pain and low back pain in relation to functional disability in different sport activities. J Exerc Rehabil 2018;14:509-515.

Ferrari S, Manni T, Bonetti F, Villafañe JH, Vanti C. A literature review of clinical tests for lumbar instability in low back pain: validity and applicability in clinical practice. Chiropr Man Therap 2015;23:14.

Henschke N, Maher CG, Refshauge KM, Herbert RD, Cumming RG, Bleasel J, York J, Das A, McAuley JH. Prognosis in patients with recent onset low back pain in Australian primary care: inception cohort study. BMJ 2008;337:a171.

Hestbaek L, Leboeuf-Yde C, Manniche C. Low back pain: what is the longterm course? A review of studies of general patient populations. Eur Spine J 2003;12:149-165.
Hodges PW. Core stability exercise in chronic low back pain. Orthop Clin North Am 2003;34:245-254.

Isgro M, Buraschi R, Barbieri C, Baruzzi E, Imperio G, Noro F, Villafañe $\mathrm{JH}$, Negrini S. Conservative management of degenerative disorders of the spine. J Neurosurg Sci 2014;58(2 Suppl 1):73-76.

Kuukkanen T, Mälkiä E. Effects of a three-month therapeutic exercise programme on flexibility in subjects with low back pain. Physiother Res Int 2000;5:46-61.

Langella F, Villafañe JH, Damilano M, Cecchinato R, Pejrona M, Ismael M, Berjano P. Predictive accuracy of surgimap surgical planning for sagittal imbalance: a cohort study. Spine (Phila Pa 1976) 2017;42:E12971304.

Lee JS, Kang SJ. The effects of strength exercise and walking on lumbar function, pain level, and body composition in chronic back pain patients. J Exerc Rehabil 2016;12:463-470.

Maniadakis N, Gray A. The economic burden of back pain in the UK. Pain 2000;84:95-103.

Moffroid MT. Endurance of trunk muscles in persons with chronic low back pain: assessment, performance, training. J Rehabil Res Dev 1997; 34:440-447.

Mousavi SJ, Parnianpour M, Mehdian H, Montazeri A, Mobini B. The Oswestry Disability Index, the Roland-Morris Disability Questionnaire, and the Quebec Back Pain Disability Scale: translation and validation studies of the Iranian versions. Spine (Phila Pa 1976) 2006;31:E454-459.

Negrini S, Imperio G, Villafañe JH, Negrini F, Zaina F. Systematic reviews of physical and rehabilitation medicine Cochrane contents. Part 1. Disabilities due to spinal disorders and pain syndromes in adults. Eur J Phys Rehabil Med 2013;49:597-609.

Nourbakhsh MR, Arab AM. Relationship between mechanical factors and incidence of low back pain. J Orthop Sports Phys Ther 2002;32:447-460.

Page SJ, Shawaryn MA, Cernich AN, Linacre JM. Scaling of the revised Oswestry low back pain questionnaire. Arch Phys Med Rehabil 2002; 83:1579-1584.

Parazza S, Vanti C, O’Reilly C, Villafañe JH, Tricás Moreno JM, Estébanez De Miguel $\mathrm{E}$. The relationship between cervical flexor endurance, cervical extensor endurance, VAS, and disability in subjects with neck pain. Chiropr Man Therap 2014;22:10.

Philips HC, Grant L. The evolution of chronic back pain problems: a longitudinal study. Behav Res Ther 1991;29:435-441.

Pillastrini P, Bonfiglioli R, Banchelli F, Capra F, Resende Fde L, Villafane $\mathrm{JH}$, Vanti C, Violante FS. The effect of a multimodal group programme in hospital workers with persistent low back pain: a prospective observational study. Med Lav 2013;104:380-392.

Pillastrini P, de Lima E Sá Resende F, Banchelli F, Burioli A, Di Ciaccio E, Guccione AA, Villafañe JH, Vanti C. Effectiveness of global postural 
re-education in patients with chronic nonspecific neck pain: randomized controlled trial. Phys Ther 2016;96:1408-1416.

Pillastrini P, Ferrari S, Rattin S, Cupello A, Villafañe JH, Vanti C. Exercise and tropism of the multifidus muscle in low back pain: a short review. J Phys Ther Sci 2015;27:943-945.

Riddle DL, Rothstein JM, Lamb RL. Goniometric reliability in a clinical setting. Shoulder measurements. Phys Ther 1987;67:668-673.

Rubin DI. Epidemiology and risk factors for spine pain. Neurol Clin 2007; 25:353-371.

Vanti C, Conti C, Faresin F, Ferrari S, Piccarreta R. The relationship between clinical instability and endurance tests, pain, and disability in nonspecific low back pain. J Manipulative Physiol Ther 2016;39:359368.

Vanti C, Ferrari S, Berjano P, Villafañe JH, Monticone M. Responsiveness of the bridge maneuvers in subjects with symptomatic lumbar spondylolisthesis: A prospective cohort study. Physiother Res Int 2017; 22(4):e1682.

Villafañe JH, Gobbo M, Peranzoni M, Naik G, Imperio G, Cleland JA, Negrini S. Validity and everyday clinical applicability of lumbar muscle fatigue assessment methods in patients with chronic non-specific low back pain: a systematic review. Disabil Rehabil 2016;38:1859-1871.

Villafañe JH, Zanetti L, Isgrò M, Cleland JA, Bertozzi L, Gobbo M, Negrini S. Methods for the assessment of neuromotor capacity in non-specific low back pain: Validity and applicability in everyday clinical practice. J Back Musculoskelet Rehabil 2015;28:201-214.

Yoon KS, Park SD. The effects of ankle mobilization and active stretching on the difference of weight-bearing distribution, low back pain and flexibility in pronated-foots subjects. J Exerc Rehabil 2013;9:292-297. 